\title{
DISEÑO DE ESTRATEGIAS DE CROWDSOURCING EN SISTEMAS DE INFORMACIÓN GEOGRÁFICA VOLUNTARIA
}

\author{
CROWDSOURCING STRATEGIES DESIGN IN VOLUNTEERED GEOGRAPHIC INFORMATION SYSTEMS \\ José-Pablo Gómez-Barrón`, Miguel-Ángel Manso-Callejo, Ramón Alcarria \\ E.T.S.I. en Topografía, Geodesia y Cartografía, Universidad Politécnica de Madrid, Km 7.5 de la Autovía de Valencia (Campus Sur), \\ 28031, Madrid, España. josepablo.gomezbarron@upm.es; m.manso@upm.es; ramon.alcarria@upm.es
}

\begin{abstract}
:
This work addresses voluntary geographic information (VGI) as an information system that facilitates organizations to achieve specific goals by outsourcing processes and activities to an online community. A definition of a voluntary geographic information system (VGIS) is proposed, identifying its core components (Project, Participants, Technology), then, crowdsourcing, the most relevant process for managing information within these type of systems, is analysed. We analyse several types of crowdsourcing models in the context of VGIS, and it is proposed a classification built around the different ways of organizing a community, which include different levels of participation according to the use of three processes: contributory, collaborative and participatory. Based on the study of the different typologies intrinsically linked to the existing levels of involvement and engagement, and the use of participants' cognitive skills, a continuum of participation is identified, presenting two opposite tendencies when designing VGI projects: crowd-based and community-driven, the latter with higher levels of collaboration or even co-creation. Based on the above, it is proposed a set of criteria for the design of the crowdsourcing strategy of a VGIS, as a roadmap that directs the project. This design and planning tool helps to characterize and define in a simple way the general requirements of the processes and activities of a VGIS that will be implemented through a crowdsourcing task, being the first step in the interdependent design of the project, participation and technological components. The design of subsequent strategies related to the other components of the system must be aligned and linked to the crowdsourcing strategy, and altogether will guide the development of tasks, functionalities and the specific technological tools of the system.
\end{abstract}

Key words: Volunteered Geographical Information, Crowdsourcing, Information Systems, Participation, Community

\section{Resumen:}

Este trabajo de investigación aborda la información geográfica voluntaria (IGV) como un sistema de información que facilita a las organizaciones el logro de objetivos específicos mediante la externalización de procesos y actividades a una comunidad en línea. En él se propone una definición de sistema de información geográfica voluntaria (SIGV), se identifican sus componentes centrales (Proyecto, Participantes, Tecnología), y se analiza el proceso de gestión de la información más relevante en este tipo de sistemas: el crowdsourcing. Se analizan varios tipos de modelos de crowdsourcing en el contexto de los SIGV, para luego proponer una clasificación basada en los diferentes modos de organizar a una comunidad; éstos incluyen distintos niveles de participación según el uso de tres procesos: contributivo, colaborativo y participativo. En base al estudio de las diferentes tipologías vinculadas intrínsecamente a los niveles de implicación y compromiso existentes, y al uso de las habilidades cognitivas de los participantes, se identifica un continuo de participación, presentándose dos tendencias opuestas al diseñar proyectos de IGV: los basados en la crowd («la multitud»), y los impulsados por una comunidad con mayor nivel de colaboración o hasta la co-creación. Basado en lo anterior, se propone un conjunto de criterios para el diseño de la estrategia de crowdsourcing de un SIGV, para dirigir un proyecto. Esta herramienta de diseño y planificación ayuda a caracterizar y definir de un modo simple los requerimientos generales de los procesos y actividades de un SIGV. Éstos serán implementados mediante una tarea de crowdsourcing, siendo el primer paso en el diseño interdependiente del proyecto, la participación y el componente tecnológico. Las estrategias a diseñar de los demás componentes del sistema se deben alinear y vincular con la estrategia de crowdsourcing, y en su conjunto guiarán el desarrollo de las tareas, funcionalidades y herramientas tecnológicas concretas del sistema.

Palabras clave: Información Geográfica Voluntaria, Crowdsourcing, Sistemas de Información, Participación, Comunidad

\section{Introducción}

Los avances en las tecnologías de la información ( $\mathrm{TI})$, particularmente el desarrollo de la Web 2.0 y la computación social centrada en las personas, han transformado Internet en una plataforma interactiva y abierta que permite la participación directa de los usuarios en la generación, manejo e intercambio de información. La Web 2.0 ha creado nuevos canales para que los usuarios en Internet no sólo actúen como consumidores pasivos de información, sino también como productores, convirtiéndose en «prosumers»

‘Corresponding Author: José-Pablo Gómez-Barrón, josepablo.gomezbarron@upm.es 
(Toffler 1980) o «produsers» (Bruns 2006), términos traducidos como «prosumidores» y «produsuarios», con un doble rol (consumidor/productor) y cuya información generalmente se denomina como «contenido generado por el usuario». Las características de interactividad, apertura, interoperabilidad y la arquitectura orientada a servicios de la Web 2.0 (O'Reilly 2005), junto a las tecnologías que ofrecen mapas digitales dinámicos, servicios web de carácter geográfico y basados en la localización, han dado lugar al desarrollo de la Geoweb o web geoespacial (Maguire 2006; Scharl 2007). En la Geoweb, Internet se utiliza para la publicación e intercambio de información geográfica (IG) y mapas (Haklay et al. 2008), y actúa como una plataforma colectiva para producir, analizar y compartir datos georreferenciados. Así, gracias a la Geoweb junto con el desarrollo y uso de los sistemas de posicionamiento por satélite, receptores GNSS (del inglés, Global Navigation Satellite Systems) y otros sensores implementados en los dispositivos móviles, imágenes de satélite, algoritmos de geocodificación y geo-tagging, entre otras herramientas que facilitan la vinculación de datos a una ubicación (localización geográfica o lugar particular), la generación de contenido geográfico en la Web por usuarios no necesariamente profesionales es cada vez mayor en la actualidad.

El panorama anterior ha reconfigurado las líneas tradicionales de demanda y oferta, así como el número de actores involucrados, en donde la sociedad en general está haciendo un uso más amplio y abierto de la geoinformación, cuando tradicionalmente estaba restringido a los sectores públicos y privados mediante la implementación de sistemas de información geográfica (SIG). Esta manipulación de la IG basada en la Web y centrada en las personas ha sido descrita por conceptos tales como «Wikification of GIS» (Sui 2008), «Mapas 2.0» (Crampton 2009) y «Neogeografía» (Turner 2006), los cuales enfatizan un nuevo paradigma de interacción entre las personas, la geografía y la creación de mapas mediante el uso diario de tecnologías geoespaciales, y las técnicas y herramientas de la Web 2.0 para descubrir, consumir, crear y compartir información, de forma más cercana a intereses individuales, incluyendo percepciones y experiencias locales propias. Sin embargo, probablemente el término que se utiliza con mayor frecuencia es el de información geográfica voluntaria (IGV). Este término focaliza en la producción de geoinformación por individuos en la Web como un caso especial de contenido generado por el usuario (Goodchild 2007a). La IGV es creada por un gran número de voluntarios generalmente no profesionales y cuya temática o dominio cubre una muy variada gama de tipos de datos valiosos en los que el espacio y el tiempo son a veces sus únicos unificadores contextuales (Elwood 2008b; Goodchild 2007b; Elwood 2008a). Esto último, presenta la oportunidad para la convergencia de los datos y de las diferentes contribuciones mediante la implementación de sistemas de información orientados a la consecución de objetivos organizacionales específicos. La IGV como actividad organizada con un propósito común, y llevada a cabo mediante un proceso consensuado que hace posible la agregación de los datos dispersos proporcionados por una comunidad en línea, utiliza el modelo conocido como crowdsourcing. Este modelo, basado en una convocatoria abierta, se emplea por las organizaciones para diseñar un proceso que externaliza la realización de una tarea a una red amplia e indefinida de individuos («la multitud» o crowd) mediante el uso de un sistema web (Howe 2006). En general, el crowdsourcing se utiliza en la adquisición de datos, procesamiento de información, generación de conocimiento, ideas y la resolución de problemas por medio de la participación abierta distribuida. La infraestructura de participación facilitada por este proceso permite a las organizaciones emplear la inteligencia y actividad colectiva de los voluntarios que optan por participar, involucrando y cooperando así con una comunidad para el logro de un objetivo organizacional, mientras que se le otorga a cambio algún tipo de beneficio. En este sentido, la IGV puede ser considerada como la IG que se ha obtenido por medio de un proceso de crowdsourcing, proporcionada por un amplio rango de participantes con diferentes niveles de educación, conocimientos y habilidades (Haklay et al. 2014).

Este artículo pretende analizar y describir los componentes involucrados en los sistemas sociotecnológicos que facilitan la tarea de producción y gestión de información geográfica voluntaria, teniendo como objetivo principal el proponer un conjunto de criterios para el diseño de las estrategias de crowdsourcing utilizadas en este tipo de sistemas. En la siguiente Sección 2, se aborda el estudio de la IGV como un sistema de información, y se propone una definición. En la Sección 3, se analiza el proceso de crowdsourcing y se propone una guía para el diseño de la estrategia a seguir, al planificar un proyecto de IGV. Finalmente, en la Sección 4, se concluyen algunas implicaciones generales en el diseño de sistemas de IGV.

\section{Sistemas de Información Geográfica Voluntaria}

Las iniciativas cuya meta es la producción o gestión de geoinformación mediante una comunidad de voluntarios, presentan varios elementos interdependientes que hacen necesario su estudio desde una perspectiva o enfoque sistémico. Esto ayuda a entender las partes de un entorno que conduce a la creación de diferentes productos o servicios de información, al igual que facilita su análisis y diseño. Como Fast and Rinner (2014) destacan, la mayoría de las investigaciones analizan las implicaciones de la IGV como un producto o conjunto de datos finales (calidad, fiabilidad, ámbitos de aplicación, etc.), sin identificar claramente los componentes y procesos existentes que influyen en su producción; y por lo tanto en la capacidad de lograr los objetivos de una organización que lleva a cabo un proyecto de IGV. En general, un sistema es un conjunto de elementos o componentes interrelacionados que trabajan conjuntamente para alcanzar un objetivo común. En este sentido, las organizaciones implementan distintos sistemas, entre los cuales se utilizan los sistemas de información (SI) como un recurso que facilita sus actividades y tareas de operación y gestión. Alter (2008), apoyándose en el concepto más general de sistema de trabajo, define sistema de información como un sistema en el cual participantes humanos y/o máquinas realizan un trabajo (procesos y actividades) utilizando información, tecnología y otros recursos para producir productos y/o servicios de información para 
clientes (personas que reciben y utilizan lo que el sistema produce) internos o externos. Las actividades y procesos que se realizan en dichos sistemas son dedicadas al procesamiento de información, lo cual implica un conjunto de procedimientos para la captura, transmisión, almacenamiento, recuperación, manipulación y visualización de la información (Alter 2008; Avison et al. 1990). Siguiendo la definición anterior se pueden identificar los componentes centrales de los SI, como: procesos y actividades, participantes (humanos), información (datos), y tecnología. También, los sistemas tienen una frontera claramente definida y existen como componentes o subsistemas de otros sistemas (existiendo vinculación entre ellos), es decir su entorno; siendo en este caso de manera inmediata la organización que planifica, diseña, desarrolla e implementa el sistema, incluyendo su visión, misión, normas, valores (cultura) y demás actividades y aspectos organizacionales que guían e influyen el rendimiento del sistema y el logro de sus objetivos. La organización muchas veces implementa un SI mediante la definición y ejecución de un proyecto, utiliza la infraestructura de que dispone (recursos humanos, informacionales y técnicos; como los equipos de trabajo y su «know-how», bases de datos y redes, etc.), y diseña las estrategias (opciones, decisiones, lógica y justificación detrás el cómo proceder) que guían el funcionamiento y operación general del sistema.

Construyendo sobre la definición de Alter (2008), en el contexto de una organización, definimos a los sistemas de información geográfica voluntaria (SIGV) como un caso especial de SI para producir productos y/o servicios de información para clientes (usuarios y «produsuarios») internos o externos mediante un proceso de crowdsourcing, aprovechando el potencial de una comunidad en línea (crowd o red distribuida de voluntarios). La comunidad en línea en este caso participa ejecutando una tarea particular de manera distribuida. Así, el trabajo central en los SIGV es realizado por la comunidad, esto quiere decir que, los procesos y actividades en estos sistemas dependen de las contribuciones de los participantes para transformar y procesar información existente y/o producir nueva información o conocimientos con un atributo geoespacial. El definir y caracterizar a los SIGV ayuda a tener un mayor entendimiento de sus componentes y atributos principales, así como sus interacciones. Esto finalmente facilita su diseño y operación de forma ordena y sistemática. Buscando simplificar el estudio, diseño e implementación de los SIGV, es posible definir como sus componentes principales, los siguientes tres elementos: Proyecto (organización), Participantes (voluntarios) y Tecnología. El proyecto implementado por la organización, incluye la definición de objetivos y la implementación de la estrategia de crowdsourcing más eficiente para su consecución. La estrategia de crowdsourcing, al ser el proceso principal del sistema, va a determinar el nivel de participación, la arquitectura de cooperación, y las características de los datos y contribuciones (información) requeridas, así como los requisitos y el uso de la tecnología que de soporte y facilite la participación y la implementación del sistema en general. Por lo tanto, el modelo de crowdsourcing desempeña un papel importante y central en el diseño y desarrollo de los SIGV, al ser un proceso que funciona como infraestructura de enlace para vincular los objetivos del proyecto y el propósito de la organización, la estructura de participación y el uso o desarrollo de diferentes herramientas tecnológicas. Actúa como una interface central para gestionar las entradas del sistema, procesar y obtener las salidas, y dirigir su funcionamiento y desempeño de forma global. En la siguiente sección se presenta un breve análisis del modelo de crowdsourcing en el contexto de los SIGV, y se introducen una serie de criterios o directrices para el diseño general de las posibles estrategias a seguir.

\section{Directrices para el Diseño de Estrategias de Crowdsourcing en SIGV}

Como se señaló en la sección anterior, con el apoyo de los sistemas de información geográfica voluntaria, las organizaciones (compañías o firmas, entidades sin ánimo de lucro, comunidades o iniciativas ciudadanas auto-organizadas, agencias del gobierno, instituciones académicas y de investigación, iniciativas de ciencia ciudadana, etc.), desarrollan e implementan productos y/o servicios de información que responden a la identificación de un área de oportunidad o problema que resolver mediante el trabajo organizado de una comunidad (principalmente online, aunque muchas veces hibrida: online/offline). El proceso y las tareas de crowdsourcing, relacionadas generalmente con la recolección, procesamiento y distribución de datos e información geográfica, así como las actividades de adquisición y gestión de conocimiento espacial y la resolución (espacial) de problemas, responden a la definición de objetivos concretos que tienen el fin último de generar algún tipo de valor. Los objetivos de un proyecto de IGV pueden incluir desde la obtención de datos de forma pasiva o contribuciones puntuales para la actualización de bases de datos, la mejora de productos o servicios de los sectores públicos y privados; pasando por actividades más activas y colaborativas como el mapeo en crisis para emergencias y respuesta humanitaria, o la investigación siguiendo un enfoque de ciencia ciudadana; hasta actividades totalmente participativas como la planificación territorial de una comunidad mediante la propuesta de ideas y soluciones creativas puestas en común entre todos los participantes. Dependiendo de los objetivos particulares, las contribuciones tienden a ser más homogéneas o heterogéneas; pasivas 0 activas; realizadas de forma individual y en muchas ocasiones aisladas $e$ independientes de las contribuciones de otros voluntarios, o basadas en una participación más colaborativa que puede llevar a la creación de un valor emergente y dependiente de las demás contribuciones. Lo anterior involucra diferentes niveles de compromiso e implicación, grados de interacción entre voluntarios, complejidad de las contribuciones y diferente grado de esfuerzo para realización de una tarea.

El propósito general de la organización, el porqué del proyecto y su objetivo claramente definido (visión), determinan el cómo dirigir al proyecto (estrategia), condicionando la planificación y diseño de las estrategias de crowdsourcing potenciales, al igual que las estrategias para atraer, fomentar y facilitar la participación como principal condicionante de éxito. A su vez, estas estrategias definirán el qué hacer (producto), es decir, qué tareas, funcionalidades, herramientas tecnológicas, etc. concretas se van a diseñar e 
implementar para probar la utilidad de las estrategias. Esto hace necesario la experimentación, adaptación y rediseño iterativo del producto, y del proyecto y sistema en su totalidad. En la siguiente subsección, con el objetivo de avanzar hacia la planificación y diseño de estrategias, se introducen diferentes enfoques generales de crowdsourcing que nos ayudan a establecer la dirección a seguir al planificar un proyecto de IGV.

\subsection{Modelos de crowdsourcing para la planificación de estrategias en proyectos de IGV}

El modelo de crowdsourcing se basa en un proceso que consiste en la obtención de recursos dispersos en una comunidad, y la agregación y asimilación de sus contribuciones utilizando herramientas web. El proceso se lleva a cabo mediante el diseño de una o varias tareas que implican el uso de las habilidades cognitivas y perceptivas de un grupo distribuido de voluntarios, participando en actividades de gestión de información y conocimiento, la resolución de problemas o generación de ideas. Brabham (2013) propone la siguiente tipología basada en los diferentes problemas para los que un determinado método de crowdsourcing es más adecuado para su resolución, identificando cuatro tipos distintos: 1. "Knowledge Discovery and Management" («Búsqueda de información y gestión de conocimiento»): Consiste en encontrar y reunir datos, información y conocimiento disperso, de una manera coherente y en un formato común; un ejemplo puede ser el realizar un reporte sobre un accidente de tráfico. 2. «Distributed Human Intelligence Tasking» («Tareas distribuidas basadas en la inteligencia humana»): Adecuado para el procesamiento y análisis de información; por ejemplo, el identificar un elemento en una imagen de satélite. 3. "Broadcast Search" («Difusión de problemas para su resolución»): ideal para investigar 0 resolver problemas con soluciones comprobables empíricamente; por ejemplo, un problema científico. 4. «Peer-Vetted Creative Production» («Producción de soluciones creativas en base al examen colectivo»): Usado para generar y seleccionar soluciones e ideas creativas o innovadoras; por ejemplo, el diseño participativo de una zona pública. Esta clasificación, nos lleva a identificar diferencias en cuanto a la dificultad de las tareas y la implicación intelectual requerida para resolver un problema, siendo más demandante a medida que nos acercamos al último método. Este último punto también se puede identificar en el análisis realizado por Haklay (2013) sobre crowdsourcing y ciencia ciudadana (geográfica) en el contexto de los proyectos de IGV. En dicho trabajo se presenta una tipología basada en cuatro niveles de participación, en los cuales el uso de las habilidades cognitivas de los voluntarios se puede considerar una variable principal. En el primer nivel propuesto, identificado simplemente como "Crowdsourcing", las exigencias cognitivas son pocas o mínimas, y se centra en la perspectiva de "citizens as a sensors» («ciudadanos como sensores humanos»), así como en el uso de "volunteering computing" o la "computación (distribuida) voluntaria». El segundo nivel, nombrado como «Distributed Intelligence» («Inteligencia Distribuida»), se asocia con el concepto de «volunteered thinking» o «pensamiento distribuido voluntario», e implica a los ciudadanos como intérpretes básicos de información siendo las habilidades cognitivas de los participantes el principal recurso del que se hace uso. El siguiente nivel se describe con el término «Participatory Science» («Ciencia Participativa») y está relacionado con la ciencia ciudadana o ciencia comunitaria, se requiere un compromiso y uso de las habilidades cognitivas de los participantes todavía mayor, para colaborar junto con científicos en la definición del problema, los métodos de recolección de datos y apoyar en el análisis a los científicos profesionales. Finalmente, se llega el término «Extreme Citizen Science» («Ciencia Ciudadana Extrema»), en este último nivel se presenta una actividad completamente participativa, desde la definición del problema, los métodos de recolección y la adquisición de datos, hasta el procesamiento de información y generación de conocimiento (incluyendo la divulgación científica) en la que tanto científicos profesionales como no profesionales tienen el papel de expertos; abriéndose también la posibilidad de hacer trabajo comunitario totalmente integrado sin la necesidad de involucrar profesionales. A medida que avanzamos en esta tipología, los proyectos requieren mayores niveles de participación, compromiso e implicación, así como pueden ir aumentando el uso de las capacidades cognitivas de los voluntarios. También, los niveles de control compartido para dirigir un proyecto, entre los iniciadores y la comunidad van adquiriendo diferentes configuraciones, apareciendo diferentes niveles de autonomía de los participantes, hasta el grado de presentarse un proyecto con casi total dependencia en una comunidad bien organizada e interdependiente para su éxito. De forma general, los proyectos que se enfocan exclusivamente en la recolección de datos, requieren menos implicación cognitiva y menor o nula colaboración entre los voluntarios, particularmente cuando estos proyectos dependen de crowdsourcing pasivo que se beneficia de los recursos tecnológicos de los dispositivos móviles digitales sin necesidad o poca intervención humana. Por el contrario, los proyectos basados en la interacción de la comunidad, por ejemplo, con el objetivo de generar conocimientos o ideas, presentan mayores niveles de colaboración y requieren mayor uso de habilidades cognitivas, las cuales son necesarias para analizar e interpretar datos y generar conocimiento y soluciones basadas en información. Estos últimos, también dependen y necesitan de la existencia de entornos más colaborativos.

En términos generales, proponemos diferenciar a los métodos de crowdsourcing en el contexto de los SIGV mediante su clasificación en distintos modos de organizar a la comunidad de participantes. Estos modos de organización de la comunidad se diferencian en que la participación puede seguir un proceso contributivo, colaborativo o participativo; los cuales difieren en el nivel de implicación y compromiso de los voluntarios, correspondiéndose también con un conjunto de diferentes motivos para participar. La distinción propuesta puede ser modelada como un continuo desde contribuciones relativamente pasivas, hacia cada vez mayores niveles de contribución activa, hasta llegar a contribuciones que necesiten de acciones proactivas. En el primer extremo del continuo se localizan los proyectos de carácter contributivo con una participación más básica sin mucha complejidad, principalmente autónoma dónde las actividades se realizan de forma independiente al trabajo de otros voluntarios. Después, 
para obtener contribuciones más complejas se requiere fomentar la comunicación y las relaciones dentro del grupo de participantes, y que, de esta manera, un proyecto empiece a operar de forma más colaborativa. Finalmente, en el otro extremo, el nivel más activo de compromiso e implicación es mediante proyectos que propician procesos participativos, permitiendo a sus participantes el estar totalmente involucrados en la decisión sobre cómo se llevará a cabo el proyecto y hasta en definir o redefinir los resultados requeridos 0 esperados, al tener un mayor peso las necesidades de información y objetivos buscados por la comunidad. Todos los métodos de crowdsourcing se basan en la contribución de una comunidad, pero no todos necesariamente requieren la colaboración 0 la implementación de un proceso participativo. En la Figura 1 se ilustran los tres niveles principales propuestos y sus procesos o modos de organización relacionados: contributivo (participación no-colaborativa), colaborativo (colaboración) y proceso participativo (co-creación).

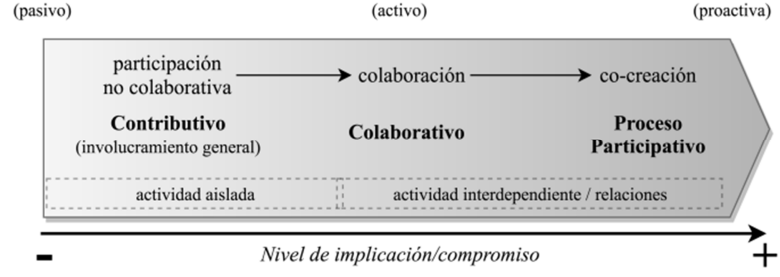

Figura 1: Niveles de participación y sus modos de organización.

En la Figura 2, se muestran como una referencia aproximada, algunos ejemplos de proyectos de IGV y su nivel de participación en relación al modo de organización. No considera un rango operacional totalmente definido en el cual se podría observar como algunos proyectos presentan varios modos de operar según diferentes actividades o momentos, sólo se ubica de forma puntal al proyecto dentro del nivel de participación más predominante para cada caso.

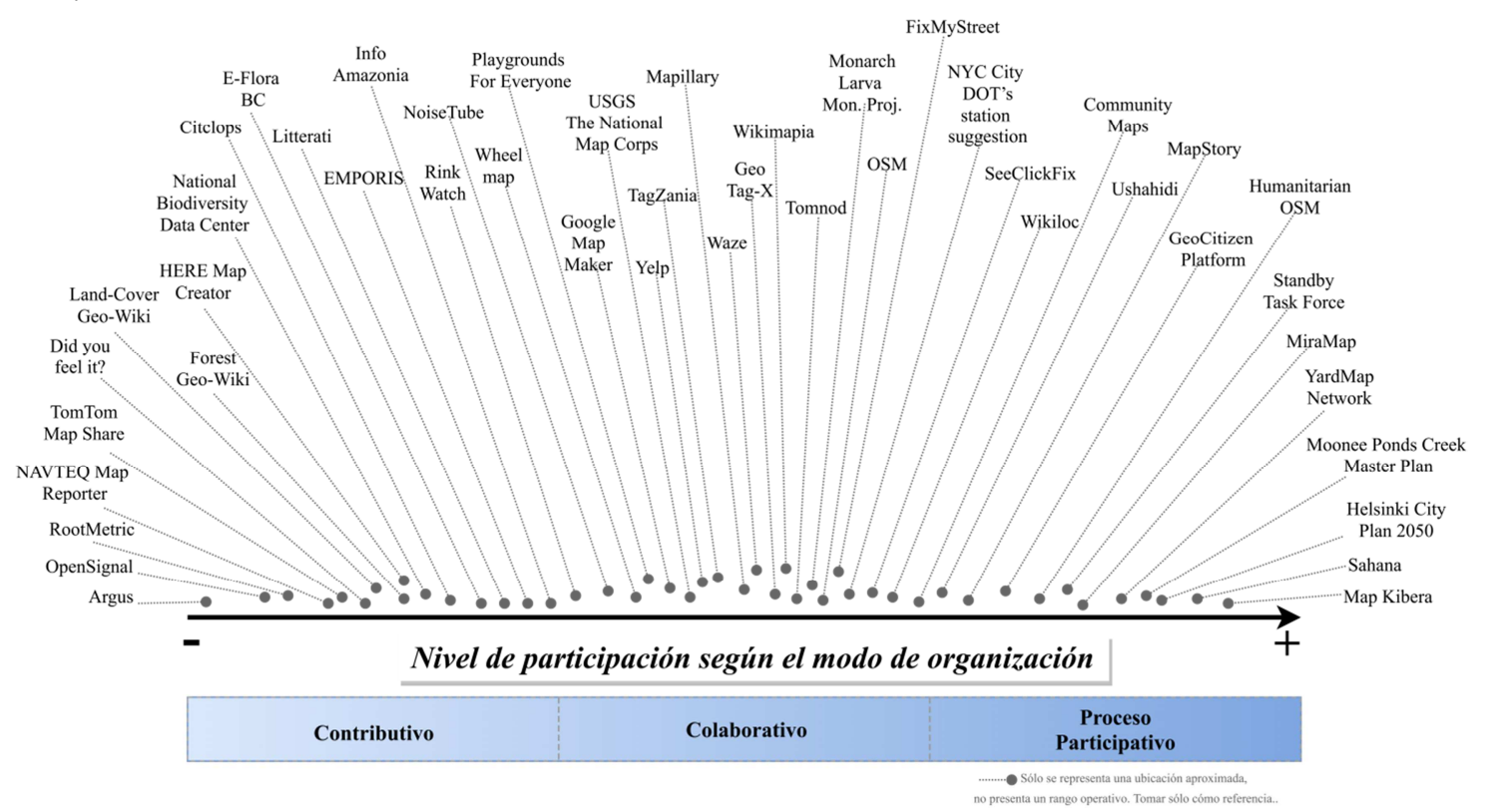

Figura 2: Proyectos IGV y su nivel de participación aproximado.

A medida que se pasa de una implicación pasiva hacia una más activa, los niveles de participación conllevan un grado creciente de interacción entre los participantes, así como con la organización (iniciadores) que gestiona el proyecto. También, niveles crecientes de colaboración hacen posible construir tareas más complejas y exigentes, por lo tanto, cuando se diseña la tarea a realizar por la comunidad es necesario considerar esta clasificación. Las tipologías introducidas anteriormente son útiles para planificar y diseñar las diferentes estrategias de crowdsourcing que se pueden utilizar por un SIGV según las características particulares del proyecto, considerándose desde el tipo de problema a resolver, el uso de las capacidades cognitivas, hasta los diferentes modos operativos de organizar a una comunidad. Se puede observar que estas tres clasificaciones de los métodos de crowdsourcing tienen puntos de inicio y finalización comunes: el primero cercano a métodos más basados en la crowd (red de individuos independientes); el segundo hacia métodos basados o manejados por la comunidad.

\subsection{Tendencias de la participación en los proyectos de IGV: crowd vs. comunidad}

Tras analizar las diferentes clasificaciones de crowdsourcing descritas, se encontró que las estrategias a seguir por un proyecto de IGV, pueden utilizar, o bien un proceso de crowdsourcing que tienda hacia una participación basada en la crowd con mayor control del proyecto por la organización, o por el contrario, una participación basada en una comunidad con mayor autonomía; al igual que pueden presentar una combinación de ambos, o que varié en el tiempo según las diferentes etapas de un proyecto. Estas estrategias opuestas, difieren en sus características y dinámicas de participación, y demandan diferentes razones, lógica o motivos por los que los voluntarios se involucran en un proyecto. Considerar estas diferencias en un proyecto de IGV es importante, ya que son factores clave para diseñar el entorno cooperativo, facilitar la participación, y el uso de métodos para atraer a diferentes tipos de voluntarios. También, el enfoque de crowdsourcing que 
sigue un proyecto, junto con su visión y objetivos, determina las características y composición de las contribuciones y los medios para obtenerlas.

En la Tabla 1 se muestran algunas de las características más importantes de la participación en línea relacionadas con cada uno de las dos tendencias del continuo de estrategias de crowdsourcing (crowd/comunidad) y su modo de operar. Para los proyectos de IGV, sirve como una guía resumida para empezar a planificar un proceso de participación basado en crowdsourcing y sus características. Estas características son las que normalmente se puede observar o esperar en cada uno de los extremos, sin embargo, en general es típico que se presente una combinación intermedia de ambas.

Tabla 1: Características de la participación en línea basada en crowd o en comunidad

\begin{tabular}{|c|c|}
\hline $\begin{array}{l}\text { Participación basada en la } \\
\text { crowd }\end{array}$ & $\begin{array}{l}\text { Participación basada en una } \\
\text { comunidad }\end{array}$ \\
\hline Contributiva & Colaborativa/Participativa \\
\hline Participación más pasiva & Participación más activa \\
\hline $\begin{array}{l}\text { Mayor control de la } \\
\text { organización }\end{array}$ & $\begin{array}{l}\text { Menor control de la } \\
\text { organización }\end{array}$ \\
\hline Menor grado de autonomía & Mayor grado de autonomía \\
\hline Individuos aislados & Asociaciones/Relaciones \\
\hline $\begin{array}{l}\text { Interacción nula/baja entre } \\
\text { participantes }\end{array}$ & $\begin{array}{l}\text { Interacción alta entre } \\
\text { participantes }\end{array}$ \\
\hline $\begin{array}{l}\text { Contribuciones } \\
\text { independientes }\end{array}$ & $\begin{array}{l}\text { Contribuciones } \\
\text { interdependientes }\end{array}$ \\
\hline $\begin{array}{l}\text { Contribuciones más } \\
\text { homogéneas }\end{array}$ & $\begin{array}{l}\text { Contribuciones más } \\
\text { heterogéneas }\end{array}$ \\
\hline $\begin{array}{c}\text { Bajo nivel de } \\
\text { implicación/compromiso }\end{array}$ & $\begin{array}{l}\text { Medio/Alto nivel de } \\
\text { implicación/compromiso }\end{array}$ \\
\hline $\begin{array}{c}\text { Tareas requieren menor } \\
\text { esfuerzo }\end{array}$ & $\begin{array}{c}\text { Tareas requieren mayor } \\
\text { esfuerzo }\end{array}$ \\
\hline $\begin{array}{l}\text { Uso de capacidades } \\
\text { cognitivas bajo }\end{array}$ & $\begin{array}{l}\text { Uso de capacidades } \\
\text { cognitivas medio/alto }\end{array}$ \\
\hline $\begin{array}{l}\text { Menor grado de } \\
\text { conocimientos/entrenamiento }\end{array}$ & $\begin{array}{c}\text { Mayor grado de } \\
\text { conocimientos/entrenamiento }\end{array}$ \\
\hline $\begin{array}{c}\text { Motivaciones individuales y } \\
\text { altruistas }\end{array}$ & $\begin{array}{l}\text { Motivaciones de grupo (en } \\
\text { formar parte de) y altruistas }\end{array}$ \\
\hline
\end{tabular}

\subsection{Diseño de la estrategia de crowdsourcing}

En esta sección se propone una herramienta para el diseño de la estrategia o proceso central de crowdsourcing de un SIGV, que será implementada en un proyecto. La estrategia es una hoja de ruta en la que se define y planifica el cómo llevar el proyecto hacia el logro de los objetivos planteados. Los objetivos particulares de un proyecto de IGV se obtienen mediante las diferentes contribuciones requeridas por los voluntarios. Para lo cual previamente, se debe identificar el modo de organización que mejor se adapte a los requisitos del flujo de trabajo a realizar por los participantes, al igual que caracterizar la tarea y sus acciones (e interacciones) centrales; lo anterior se encuentra enmarcado dentro de una estrategia de crowdsourcing. En la sección anterior analizamos diferentes tipologías de crowdsourcing que se encuentran intrínsecamente vinculadas según el nivel existente de implicación y compromiso en la participación. Las distintas características de los métodos analizados nos llevaron a identificar un espectro o continuo de participación, y definir dos tendencias existentes al diseñar proyectos: los basados en la crowd, y los impulsados por una comunidad con mayores niveles de colaboración. Relacionado con lo anterior, también observamos que, a medida que el nivel de participación aumenta, lo cual se traduce en mayor implicación y compromiso de los voluntarios, el modo de operar del proyecto tiende a pasar de sólo contribuciones rutinarias, a la colaboración y cocreación. Correspondientemente, los niveles más altos de participación, como ilustra la tipología de Haklay, también están correlacionados con un mayor uso de las capacidades cognitivas de los participantes, lo que permite diseñar y solicitar tareas que conlleven mayor esfuerzo o complejidad.

Basado en lo anterior, una vez que se ha caracterizado un proyecto y se tiene definido su objetivo, proponemos identificar y diseñar la estrategia o proceso central de crowdsourcing que requiere un SIGV usando dos criterios:

1. Nivel de participación (implicación/compromiso) según el modo de organización contributivo, colaborativo o participativo.

2. Implicación cognitiva de los participantes para realizar la tarea, en dónde proponemos su caracterización en función de la demanda cognitiva y de las habilidades requeridas para realizar la tarea.

El primer criterio, introducido inicialmente en la sección 3.1 , corresponde al nivel de participación existente en una comunidad, determinado por el modo de organización (operación) más adecuado para obtener y asimilar las contribuciones de los participantes que un proyecto requiere; los modos de operación se clasifican en contributivo, colaborativo o participativo (co-creativo). Como ya se mencionó anteriormente, los distintos niveles de implicación y compromiso de los voluntarios permiten diferentes grados de interacción, interdependencia de las contribuciones, complejidad de las tares y la posibilidad o no de generar valor emergente basado en la colaboración. El segundo criterio, la implicación cognitiva requerida de los participantes, se relaciona con el grado o cantidad de esfuerzo mental y las habilidades necesarias para trabajar en una tarea. Basado en investigaciones del dominio cognitivo (procesar información, conocimiento y habilidades mentales) y los procesos de aprendizaje, la demanda cognitiva se considera una característica intrínseca a las tareas y consiste en el tipo y nivel de pensamiento requerido para trabajar en una tarea y resolverla (Smith and Stein 1998). Las tareas de baja demanda cognitiva involucran el señalar hechos, seguir procedimientos conocidos y resolver problemas rutinarios, no requieren un gran esfuerzo intelectual y se centran principalmente en proporcionar respuestas concretas y usar conocimientos previos sin necesidad de establecer o realizar nuevas conexiones (Van De Walle et al. 2012). Por el contrario, las tareas de demanda cognitiva alta involucran el establecimiento de 
conexiones, analizar la información y sacar conclusiones (Smith and Stein 1998). Por otro lado, siguiendo a Erickson et al. (2012), podemos clasificar las habilidades necesarias para completar una tarea de crowdsourcing como: habilidades comunes o generales, requeridas en tareas sencillas y rutinarias; habilidades especializadas o avanzadas; y habilidades específicamente relacionadas a un dominio científico o tecnológico particular. Finalmente, con el objetivo de caracterizar la implicación cognitiva global o total para realizar una tarea, de menor a mayor uso de habilidades cognitivas por los participantes, proponemos tomar en consideración tanto la demanda cognitiva (clasificándola como baja, media y alta) y las habilidades requeridas para realizar la tarea (comunes, especializadas y relacionadas a un dominio). Combinando ambos criterios, podemos considerar el nivel más bajo de implicación cognitiva, cuando se requiere una demanda cognitiva baja y se hace uso de una habilidad común para realizar una tarea; por el contrario, el nivel más alto corresponde a una tarea que requiere una demanda cognitiva alta y hace uso de habilidades relacionadas con un dominio de conocimiento específico. Adicionalmente a los dos criterios principales, es de utilidad introducir la taxonomía de Bloom de habilidades de pensamiento, revisada y modificada por Anderson and Krathwohl (2001). Esta taxonomía sirve como soporte adicional al caracterizar la implicación y demanda cognitiva que tendrá una tarea relacionada con la gestión de información y la resolución de problemas, entendiendo mejor cómo abordar su diseño y las características de participación. La taxonomía revisada incluye seis categorías de habilidades de pensamiento en orden creciente de demanda cognitiva. Las habilidades de pensamiento de orden inferior son: recordar, asociada a acciones como identificar, enlistar, reconocer o describir algo; entender, relacionada a actividades como explicar, interpretar y resumir información; y aplicar, relacionada con implementar, calcular, y el uso y aplicación de información previa. En cuanto a las habilidades de pensamiento de orden superior se presentan las siguientes: analizar, asociada a comparar, integrar, encontrar e inferir información; evaluar, relacionada con la deducción y acciones como juzgar, experimentar, monitorizar y formular hipótesis; crear, que involucra acciones como producir, idear, planificar y diseñar.

El análisis de los criterios propuestos para diseñar una estrategia de crowdsourcing ayuda a formular, responder y planificar cuestiones de diseño como las siguientes: ¿Qué nivel de complejidad, demanda cognitiva y habilidades son necesarias para que los participantes puedan realizar las tareas de gestión de información que se van externalizar a la comunidad? ¿Cuál es la dinámica de participación requerida mientras se realiza una tarea y para contribuir en los diferentes proceses del proyecto? ¿La demanda cognitiva de una tarea hace necesario implementar un modo de organización específico para la comunidad, para poder llegar al resultado esperado? ¿Se tiene como objetivo generar un conocimiento emergente basado en la colaboración sinérgica? ¿Es necesaria una colaboración interdependiente $\mathrm{O}$ un proceso participativo?, o, por el contrario, ¿Es posible de lograr los objetivos mediante contribuciones aisladas? La Figura 3 ilustra visualmente los criterios de diseño propuestos para caracterizar una estrategia de crowdsourcing. También, ayuda a identificar si la participación tiene características con tendencia hacia la crowd o hacia una comunidad con mayor interacción. Además, se localizaron de forma aproximada algunos métodos de crowdsourcing y actividades relacionadas dentro del diagrama, como referencia de su funcionamiento en relación con los criterios de diseño.

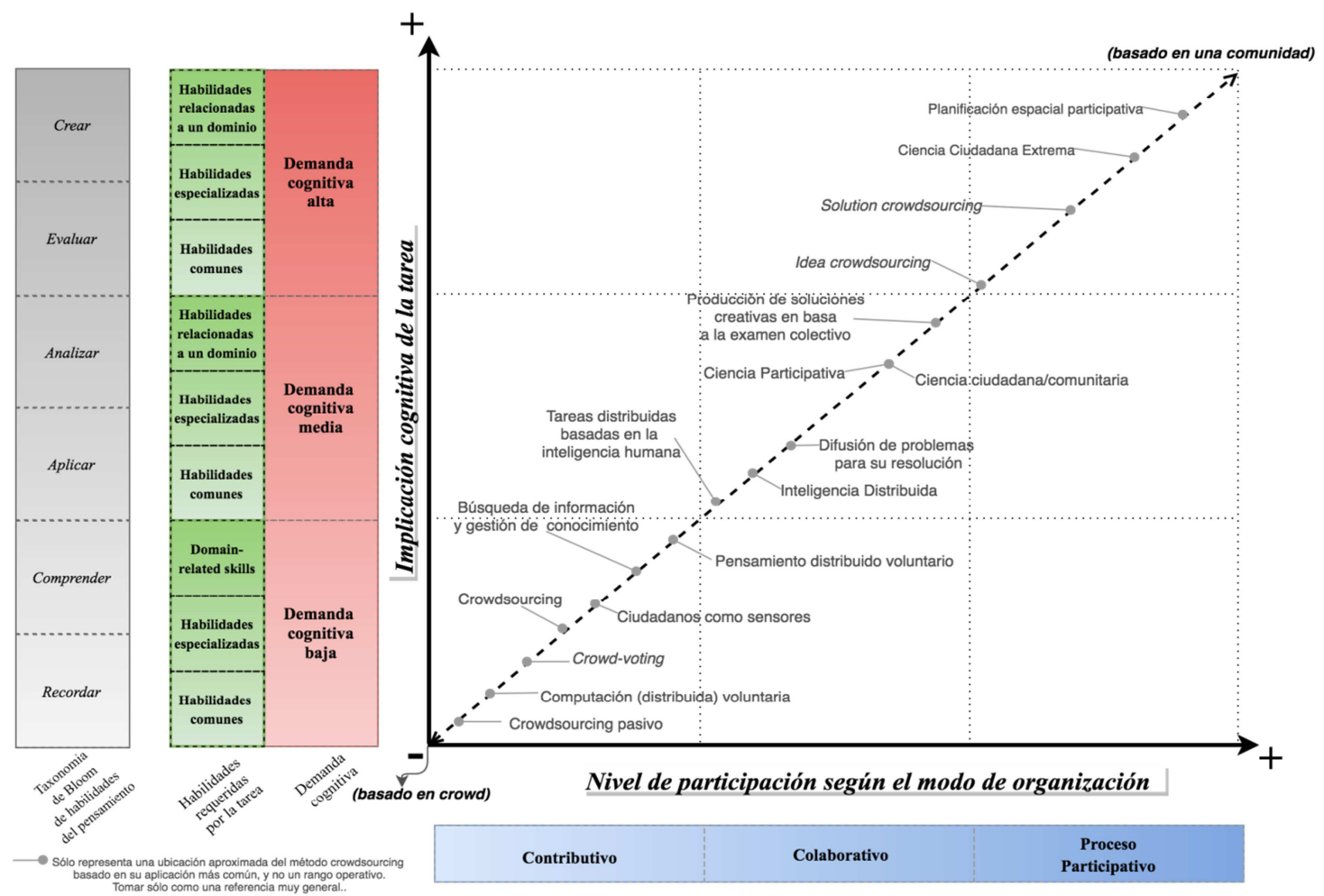

Figura 3: Criterios de diseño de la estrategia de crowdsourcing de un proyecto de IGV. 


\section{Conclusiones}

En este trabajo se abordó el diseño de una de las estrategias necesarias de definir y planificar en los SIGV, la estrategia de crowdsourcing. Esta estrategia se define dentro del contexto de un proyecto de IGV, siendo el primer paso y variable de la que depende la planificación y el diseño interdependiente de los otros componentes del sistema: participantes y tecnología. Las demás estrategias del sistema, tales como las estrategias para atraer, facilitar, sostener y retener la participación, así como las relacionadas con el desarrollo del componente tecnológico, no se han analizado en este trabajo. Sin embargo, estas últimas están totalmente vinculadas, al tratarse de un enfoque de diseño sistémico, a la estrategia de crowdsourcing, la cual debe de ser la primera en diseñarse siendo la que guie a las demás estrategias junto con otros criterios del proyecto.

Los criterios que se han propuesto en la herramienta de diseño de la estrategia de crowdsourcing, ayudan a caracterizar y definir de un modo simple los requerimientos generales de los procesos y las actividades de un SIGV que se pretenden externalizar a una red distribuida de individuos en la Web; ya sea siguiendo una actividad realizada de forma independiente por una crowd, o buscando una mayor colaboración para aprovechar los beneficios del trabajo integrado en una comunidad. Es necesario que las posteriores etapas de desarrollo e implementación del proyecto y las herramientas tecnológicas, en las que se definirá la mecánica de las tareas de crowdsourcing y sus acciones núcleo concretas, estén fundamentadas y alineadas con esta estrategia, ya que los criterios de diseño propuesto tienen un impacto directo en su desarrollo. De igual forma, al diseñar las tareas, no es posible considerar de manera independiente la estrategia de crowdsourcing (el cómo se pretende obtener las contribuciones) y la naturaleza de los datos e información de las contribuciones (incluyendo sus usos, calidad esperada o requerida y demás atributos), sino de forma integrada.

Entre las características necesarias a considerar, se encuentra la dimensión espacial de la contribución. Ésta puede ser asociada a una localización o coordenadas específicas que se basan en un marco de referencia geográfica (por ejemplo una medida u observación física o medioambiental objetiva asociada a una coordenada); o una contribución (generalmente de carácter más subjetivo) vinculada a un lugar (definido como «una localización creada por experiencias humanas» (Grantham and Tuan 1978), con características físicas y humanas asociadas), como un barrio, una calle, un centro deportivo o una iglesia. En cuanto a los atributos de la información se tendría que considerar la flexibilidad en su estructura, presentándose de forma estructurada o no estructurada (Deparday 2010), y en su caso los diferentes niveles de estructuración. También si se trata de hechos u opiniones, diferenciándose en contribuciones objetivas o subjetivas (Tulloch 2008; Deparday 2010). Y finalmente el grado de interacción en el flujo de los datos: unidireccional, bidireccional o ndireccional (Deparday 2010). Este último atributo, directamente relacionado con contribuciones individuales o la colaboración de los participantes, expuesto en secciones anteriores. Además, las herramientas tecnológicas a utilizar o desarrollar, así como las acciones de los participantes para la obtención de información y modos de captura, van estar determinadas por estas características espaciales y los diferentes tipos de atributos.

Por otro lado, en este trabajo también se han abordado los aspectos interdependientes al analizar la producción de la IGV mediante el estudio y desarrollo de un sistema. Se identificó a la organización y el proyecto, el proceso y las contribuciones basado en crowdsourcing, y su vinculación con la participación y el uso de tecnología, como elementos esenciales que hay que analizar y tomar en consideración al diseñar e implementar un SIGV. En este sentido, el diseño de un SIGV sigue una serie de pasos que involucran a sus componentes e interacción. Iniciando desde la detección de un problema u oportunidad que se puede abordar mediante el crowdsourcing de IG y la organización de una comunidad en línea, la definición de los propósitos y objetivos de un proyecto, para pasar a la exploración de las estrategias de crowdsourcing más adecuadas. Los pasos siguientes consisten en alinear y vincular los objetivos y necesidades organizacionales con la mejor estrategia de crowdsourcing (analizado en el este trabajo); basado en lo anterior, definir las características de la información a contribuir, e identificar factores que motiven y faciliten la participación según las características de la estrategia definida. Después, la vinculación y alineación de la estrategia de crowdsourcing con un entorno de cooperación adecuado (incluyendo la definición de las tareas y acciones a realizar por los participantes), y con las estrategias de participación necesarias (para atraer, enganchar y retener la participación). Por último, la vinculación con el desarrollo de las herramientas tecnológicas más adecuadas que faciliten las tareas y acciones de los participantes.

Finalmente, es importante el definir criterios y métricas de control para observar el rendimiento y resultados del sistema, incluyendo los criterios de éxito que nos indiquen si las estrategias y herramientas implementadas están funcionando para avanzar hacia el logro de los objetivos. Lo anterior, basado en las medidas de rendimiento, facilita el realizar experimentos para la mejora iterativa del sistema utilizando metodologías de desarrollo Lean y Agiles.

\section{References}

ALTER, S., 2008. Defining information systems as work systems: implications for the IS field. European Journal of Information Systems, 17(5), pp. 448-469.

ANDERSON, L.W. and KRATHWOHL, D.R., 2001. A taxonomy for learning, teaching, and assessing: A revision of Bloom's taxonomy of educational objectives. Theory Into Practice, Complete e, p.xxix, 352 . Available: http://www.mendeley.com/research/a-taxonomy-for-learning-teaching-and-assessing-a-revision-of-blooms-taxonomyof-educational-objectives-abridged-edition-1/. 
AVISON, D., WOOD-HARPER, A. and ANTILL, L., 1990. Multiview: An exploration in information systems development, Alfred Waller Ltd. Available: http://library.wur.nI/WebQuery/clc/549448 [4/25, 2016].

BRABHAM, D.C., 2013. Crowdsourcing The MIT Pr., MIT Press. Available: https://books.google.com/books?hl=es\&lr=\&id=ndcnAAAAQBAJ\&pgis=1 [9/16, 2015].

BRUNS, A., 2006. Towards Produsage: Futures for User-Led Content Production. Cultural Attitudes towards Communication and Technology.

CRAMPTON, J.W., 2009. Cartography: maps 2.0. Progress in Human Geography, 33(1), pp. 91-100.

DEPARDAY, V., 2010. Enhancing Volunteered Geographical Information ( VGI ) Visualization with Open Source WebBased Software. University of Waterloo. Available:

http://www.google.com/url?sa=t\&source=web\&cd=2\&ved=0CBwQFjAB\&url=http://uwspace. uwaterloo.ca/bitstream/10 012/5709/1/Deparday_Vivien.pdf\&ei=UgboTaWTIKLSiALNo6nSCw\&usg=AFQjCNGvZGVKSRiGprb7OoeXNnPCRFt rdQ.

ELWOOD, S., 2008a. Volunteered geographic information: Future research directions motivated by critical, participatory, and feminist GIS. GeoJournal, 72(3-4), pp. 173-183.

ELWOOD, S., 2008b. Volunteered geographic information: Key questions, concepts and methods to guide emerging research and practice. GeoJournal, 72(3-4), pp. 133-135.

ERICKSON, L.B., PETRICK, I., and TRAUTH, E.M., 2012. Hanging with the right crowd: Matching crowdsourcing need to crowd characteristics. In AMCIS 2012 Proceedings. Seattle, USA, pp. 1-9.

FAST, V. and RINNER, C., 2014. A Systems Perspective on Volunteered Geographic Information. ISPRS International Journal of Geo-Information, 3(4), pp. 1278-1292. Available: http://www.mdpi.com/2220-9964/3/4/1278/.

GOODCHILD, M.F., 2007a. Citizens as sensors: the world of volunteered geography. GeoJournal, 69(4), pp.211-221. Available: http://link.springer.com/10.1007/s10708-007-9111-y [7/14, 2014].

GOODCHILD, M.F., 2007b. Citizens as voluntary sensors: spatial data infrastructure in the world of Web 2.0 . International Journal of Spatial Data Infrastructure Research, 2, pp.24-42. Available: http://citeseerx.ist.psu.edu/viewdoc/summary?doi=10.1.1.162.2017 [4/25, 2016].

GRANTHAM, C.E. and TUAN, Y.F., 1978. Space and Place: The Perspective of Experience. Contemporary Sociology, 7(4), p.513.

HAKLAY, M., 2013. Citizen Science and Volunteered Geographic Information: Overview and Typology of Participation. In D. Sui, S. Elwood, \& M. Goodchild, eds. Crowdsourcing Geographic Knowledge. Springer Netherlands, pp. $105-122$. Available: citeulike-article-id:13292849\%5Cnhttp://dx.doi.org/10.1007/978-94-007-4587-2_7.

HAKLAY, M., ANTONIOU, V. and BASIOUKA, S., 2014. Crowdsourced Geographic Information Use in Government, Available: http://discovery.ucl.ac.uk/1433169/ [9/10, 2014].

HAKLAY, M., SINGLETON, A. and PARKER, C., 2008. Web mapping 2.0: The neogeography of the GeoWeb. Geography Compass, 2(6), pp. 2011-2039.

HOWE, J., 2006. The Rise of Crowdsourcing. Wired Magazine, 14(6), pp.1-5.

MAGUIRE, D., 2006. GeoWeb 2.0 Implications for ESDI. In 12th EC-GIGIS Workshop. Innsbruck, Austria. Available: http://www.ec-gis.org/Workshops/12ec-gis/presentations/Plenary room/Opening/GeoWeb2_2.pdf [4/25, 2016].

O’REILLY, T., 2005. What Is Web 2.0. Available: http://oreilly.com/web2/archive/what-is-web-20.html [3/6, 2017].

SCHARL, A., 2007. Towards the geospatial web: media platforms for managing geotagged knowledge repositories. In The Geospatial Web - How Geo-Browsers, Social Software and the Web 2.0 are Shaping the Network Society. pp. 3-14.

SMITH, M.S. and STEIN, M.K., 1998. Selecting and creating mathematical tasks: From research to practice. Mathematics Teaching in the Middle School, 3(5), pp.344-350.

SUI, D.Z., 2008. The wikification of GIS and its consequences: Or Angelina Jolie's new tattoo and the future of GIS. Computers, Environment and Urban Systems, 32(1), pp.1-5.

TOFFLER, A., 1980. The Third Wave,

TURNER, A., 2006. Introduction to Neogeography, O'Reilly Media, Inc. Available: http://books.google.com/books?hl=es\&lr=\&id=oHgDv4feV-8C\&pgis=1 [11/27, 2014].

VAN DE WALLE, J., KARP, K. and BAY-WILLIAMS, J., 2012. Elementary and middle school mathematics: Teaching developmentally 8th ed., Pearson. 